\title{
High Acuity Therapy Variation Across Pediatric Acute Care Cardiology Units: Results from the Pediatric Acute Care Cardiology Collaborative Hospital Surveys
}

\author{
Ashraf S. Harahsheh ${ }^{1}$ (1) . Alaina K. Kipps ${ }^{2}$. Stephen A. Hart ${ }^{3}$. Steven C. Cassidy ${ }^{3} \cdot$ Martha L. Clabby $^{4}$. \\ Anthony M. Hlavacek ${ }^{5}$. Amanda K. Hoerst ${ }^{6} \cdot$ Margaret A. Graupe $^{6}$. Nicolas L. Madsen ${ }^{6,7}$. Adnan M. Bakar ${ }^{8}$. \\ Erica L. Del Grippo ${ }^{9}$. Sonali S. Patel ${ }^{10}$. James E. Bost ${ }^{11} \cdot$ Ronn E. Tanel ${ }^{12}$ on behalf of the Pediatric Acute Care \\ Cardiology Collaborative (PAC ${ }^{3}$ )
}

Received: 26 October 2020 / Accepted: 18 March 2021 / Published online: 4 April 2021

(c) The Author(s), under exclusive licence to Springer Science+Business Media, LLC, part of Springer Nature 2021

\begin{abstract}
We utilized the multicenter Pediatric Acute Care Cardiology Collaborative $\left(\mathrm{PAC}^{3}\right) 2017$ and 2019 surveys to describe practice variation in therapy availability and changes over a 2-year period. A high acuity therapies (ATs) score was derived (1 point per positive response) from 44 survey questions and scores were compared to center surgical volume. Of 31 centers that completed the 2017 survey, 26 also completed the 2019 survey. Scores ranged from 11 to 34 in 2017 and 11 to 35 in 2019. AT scores in 2019 were not statistically different from 2017 scores (29/44, IQR 27-32.5 vs. 29.5/44, IQR 27-31, $p=0.9$ ). In 2019, more centers reported initiation of continuous positive airway pressure (CPAP) and Bi-level positive airway pressure (BiPAP) in Acute Care Cardiology Unit (ACCU) (19/26 vs. 4/26, $p<0.001)$ and permitting continuous CPAP/BiPAP (22/26 vs. $14 / 26, p=0.034$ ) compared to 2017 . Scores in both survey years were significantly higher in the highest surgical volume group compared to the lowest, $33 \pm 1.5$ versus $25 \pm 8.5, p=0.046$ and $32 \pm 1.7$ versus $23 \pm 5.5, p=0.009$, respectively. Variation in therapy within the ACCUs participating in $\mathrm{PAC}^{3}$ presents an opportunity for shared learning across the collaborative. Experience with PAC $^{3}$ was associated with increasing available respiratory therapies from 2017 to 2019 . Whether AT scores impact the quality and outcomes of pediatric acute cardiac care will be the subject of further investigation using a comprehensive registry launched in early 2019.
\end{abstract}

Keywords Pediatric cardiology $\cdot$ Congenital heart disease $\cdot$ Hospitalist medicine $\cdot$ Pediatric Acute Care Cardiology Collaborative $\left(\mathrm{PAC}^{3}\right) \cdot$ Inpatient cardiology

Ashraf S. Harahsheh

aharahsh@childrensnational.org

1 Division of Cardiology, Department of Pediatrics, Children's National Hospital, George Washington University School of Medicine \& Health Sciences, 111 Michigan Ave, NW, Washington, DC 20010, USA

2 Division of Cardiology, Department of Pediatrics, Stanford School of Medicine, Palo Alto, CA, USA

3 Division of Cardiology, Department of Pediatrics, Nationwide Children's Hospital, The Ohio State University, Columbus, $\mathrm{OH}$, USA

4 Department of Pediatrics, Children's Healthcare of Atlanta, Emory University School of Medicine, Atlanta, GA, USA

5 Division of Pediatric Cardiology, Department of Pediatrics, Medical University of South Carolina, Charleston, SC, USA

6 Heart Institute, Cincinnati Children's Hospital Medical Center, Cincinnati, OH, USA
7 Division of Pediatric Cardiology, Department of Pediatrics, University of Cincinnati School of Medicine, Cincinnati, OH, USA

8 Division of Critical Care Medicine, Department of Pediatrics, Cohen Children's Medical Center, New Hyde Park, NY, USA

9 Nemours Cardiac Center at A.I. duPont Hospital for Children, Wilmington, DE, USA

10 Department of Pediatrics, Section of Cardiology, University of Colorado School of Medicine, Aurora, CO, USA

11 Division of Biostatistics and Study Methodology, Children's National Hospital, George Washington University School of Medicine, Washington, DC, USA

12 Division of Pediatric Cardiology, Department of Pediatrics, UCSF Benioff Children's Hospital, UCSF School of Medicine, San Francisco, CA, USA 


$\begin{array}{ll}\text { Abbreviations } \\ \text { ATs } & \text { High acuity therapies } \\ \text { ACCU } & \text { Acute care cardiology unit } \\ \text { BiPAP } & \text { Bi-level positive airway pressure } \\ \text { CNU } & \text { Cardiac Networks United } \\ \text { CPAP } & \text { Continuous positive airway pressure } \\ \text { IQR } & \text { Interquartile range } \\ \text { PAC } & \text { Pediatric Acute Care Cardiology Collaborative } \\ \text { PGE1 } & \text { Prostaglandin E1 } \\ \text { REDCap } & \text { Research electronic data capture }\end{array}$

\section{Introduction}

The multicenter Pediatric Acute Care Cardiology Collaborative $\left(\mathrm{PAC}^{3}\right)$ was established in 2014 to deliver higher quality care for patients admitted to the acute care cardiology unit (ACCU) [1, 2]. Initial work from this collaborative was a survey in 2017 and repeated with similar questions in 2019 to understand unit structure, practices, and resource utilization at participating centers [3]. A detailed description of therapies available to ACCUs has not been previously described [4]. We aim to describe practice variation regarding high acuity therapies (ATs) (e.g., inotropic support) across the PAC ${ }^{3}$ member institutions, assess if AT scores changed over time, and assess AT association with center surgical volume.

\section{Methods}

The PAC ${ }^{3}$ conducted an initial member survey in 2017 assessing practice variation [3]. The survey components addressed hospital demographics, staffing, high acuity therapies and resource utilization, use of standard care practices, and patient transfer and discharge practices. The survey included 15 stem questions pertaining to AT in ACCUs, with branching questions following positive responses. They covered items related to inotropic infusions, therapies for pulmonary hypertension and arrhythmias, anticoagulation, immune therapy, vascular access, drains and dialysis, respiratory care, sedation, use of prostaglandin E1 (PGE1), anti-neoplastic agents, and ventricular assist devices. An AT score was derived (1 point for each positive response) from 44 survey questions on types of therapies offered on the ACCU. The survey data were collected and managed using Research Electronic Data Capture (REDCap) tools hosted at Cincinnati Children's Hospital Medical Center [5]. A follow up survey was conducted in 2019 to assess if therapy offerings, and thus AT scores, changed among participating centers.

\section{Statistical Analysis}

Descriptive analyses of the PAC P $^{3}$ AT scores in 2017 and 2019 were reported as median and interquartile range (IQR). AT availability is summarized using frequencies and percentages and tested between 2017 and 2019 using Fisher's exact test. The total 2019 AT scores were compared to 2017 AT scores with Wilcoxon signed rank sum test. For each AT score, "a Cronbach alpha $(\alpha)$ score was computed to assess internal consistency" [6]. The association between AT scores and center surgical volume, which was previously found to correlate with unit size, was assessed [3]. Centers were grouped according to annual surgical case volume, either self-reported or referenced from Society of Thoracic Surgery data: $\leq 300$, 301-500, 501-700, and $>700$ cases/year. The Kruskal Wallis test was used to test the AT score between surgical volume groups in 2017 and 2019 , respectively. The Dunn test was used as a post hoc analysis to perform multiple comparisons if the Kruskal Wallis test was significant. A $p$-value $<0.05$ was considered significant. Analyses were performed using SAS 9.4 software (SAS Institute, North Carolina, USA).

Waiver of consent was approved by the Institutional Review Board at Cincinnati Children's Hospital Medical Center.

\section{Results}

The 2017 PAC $^{3}$ surveys were completed by $31(91 \%)$ of the 34 member centers at the time of survey distribution. Of the 31 centers that completed the 2017 survey, 26 also completed the 2019 survey and their AT responses were included in this study (Fig. 1).

The 2017 median AT score $(\alpha=0.76)$ was 29.5 , IQR 27-31. Variation in offering of the 44 different therapies reported by each unit was observed ranging from 11 to 34 per individual center in 2017 (Fig. 2). Individual AT item availability was also noted to vary considerably. Chest tubes to suction, peripherally inserted central catheter line, intermittent dosing of intravenous narcotic pain medication, and intravenous immune globulin were universally offered, but only $2(7.7 \%)$ centers permitted use of intravenous epinephrine in the ACCU (Table 1).

The median 2019 AT score $(\alpha=0.79)$ was $29 / 44$ (IQR 27-33) and was not statistically different from 2017 AT score $(p=0.9)$. Over the 2 -year period between the two surveys, 13/26 centers had an increase in their overall AT score but $12 / 26$ had a decrease and one remained at the same AT score. We noted differences in several respiratory 
Fig. 1 Number of Centers included in this study

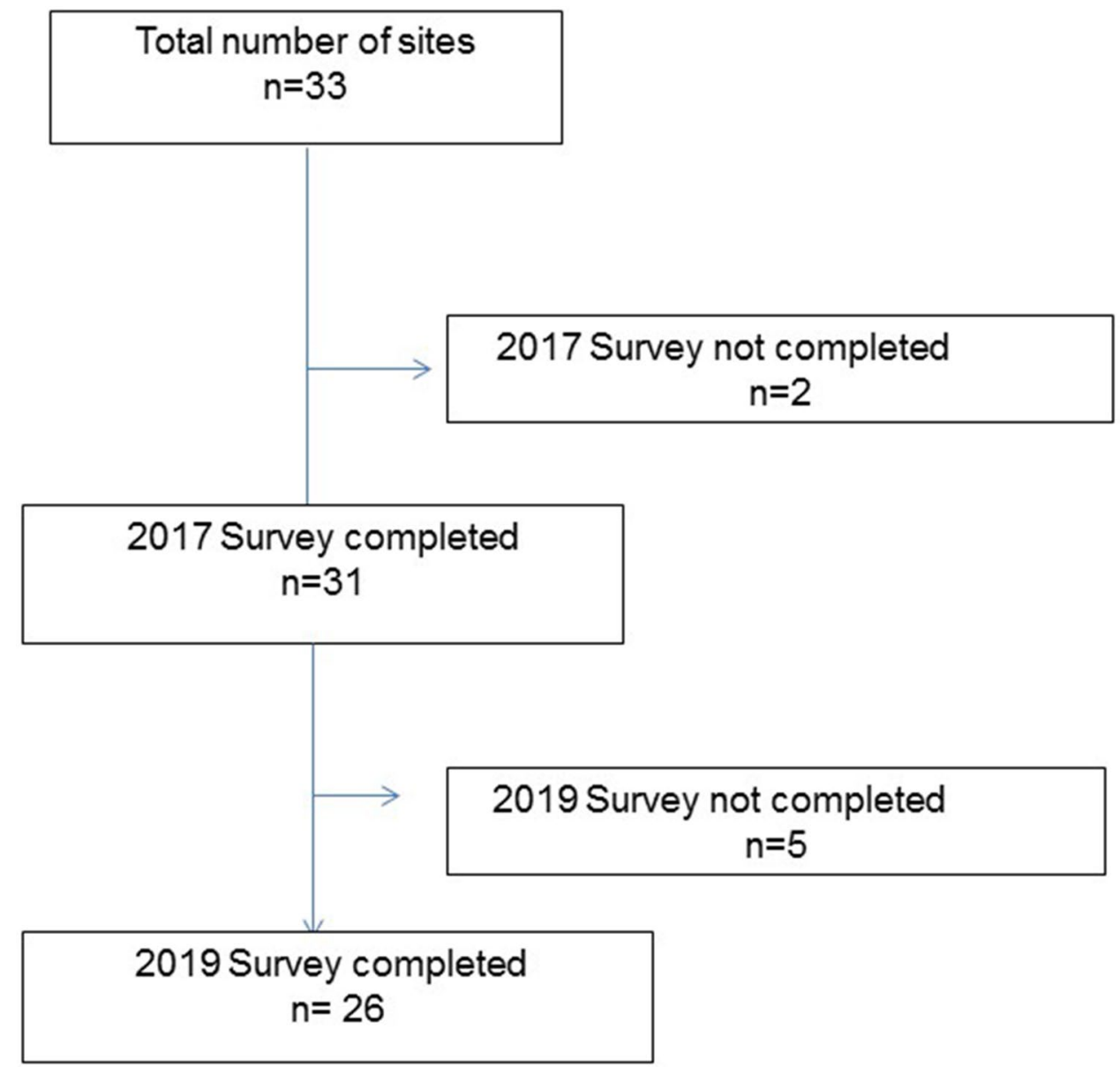

therapies offered in ACCUs among participating centers between the two surveys. More centers reported initiation of continuous positive airway pressure (CPAP) and $\mathrm{Bi}$ level positive airway pressure (BiPAP) in ACCU (19/26 vs. 4/26, $p<0.001)$ and permitting continuous CPAP/ BiPAP (22/26 vs. 14/26, $p=0.034)$ in 2019 compared to 2017. Thus, fewer centers offered CPAP/BiPAP only while sleeping in 2019 compared to 2017 surveys (3/26 vs. $21 / 26, p<0.001)$. On the other hand, less centers offered tracheostomy care with or without oxygen in 2019 compared to 2017 surveys $(11 / 26$ vs. $21 / 26, p=0.009)$. Although not statically significant, more centers reported permitting advanced tracheostomy care in 2019 than 2017 (with intermittent home ventilator requirement (e.g., night only) $14 / 26$ vs. $11 / 26, p=0.58$; or with continuous home ventilator ( $24 \mathrm{~h} /$ day) $11 / 26 \mathrm{vs.} 9 / 26, p=0.78$ ) (Table 1$)$.

The AT scores from both surveys were significantly higher in the highest surgical volume group compared to the lowest surgical volume group, $33 \pm 1.5$ versus $25 \pm 8.5$, $p=0.046 ; 32 \pm 1.7$ versus $23 \pm 5.5, p=0.009$, respectively (Fig. 3). In 2019, inhaled prostacyclin (e.g., Iloprost), ventricular assist device, peritoneal dialysis, temporary pacer wires, tracheostomy and intravenous intermittent dosing of benzodiazepines were more likely to be found among centers with highest surgical volume than among centers with lowest surgical volume (Table 2).

\section{Discussion}

There is significant variation in the types of therapy offered on units across the $\mathrm{PAC}^{3}$ collaborative institutions. This practice variation presents an opportunity for shared learning across the collaborative, a cornerstone of the PAC ${ }^{3}$ mission [1]. How practices shift over time can also reveal opportunities for collaboration to achieve quality care. While the median AT scores in the two surveys were not statistically different, in 2019 more centers reported initiation and permission of continuous CPAP/BiPAP compared to 2017. We believe that this may be in part related to shared learning across the collaborative. In the 2-year period, four in-person learning sessions occurred with devoted time to discuss practice variation. Centers would have been able to challenge limitations to therapies offered at their own site, empowered by the evidence that other centers with similar surgical volume were implementing higher therapeutic 


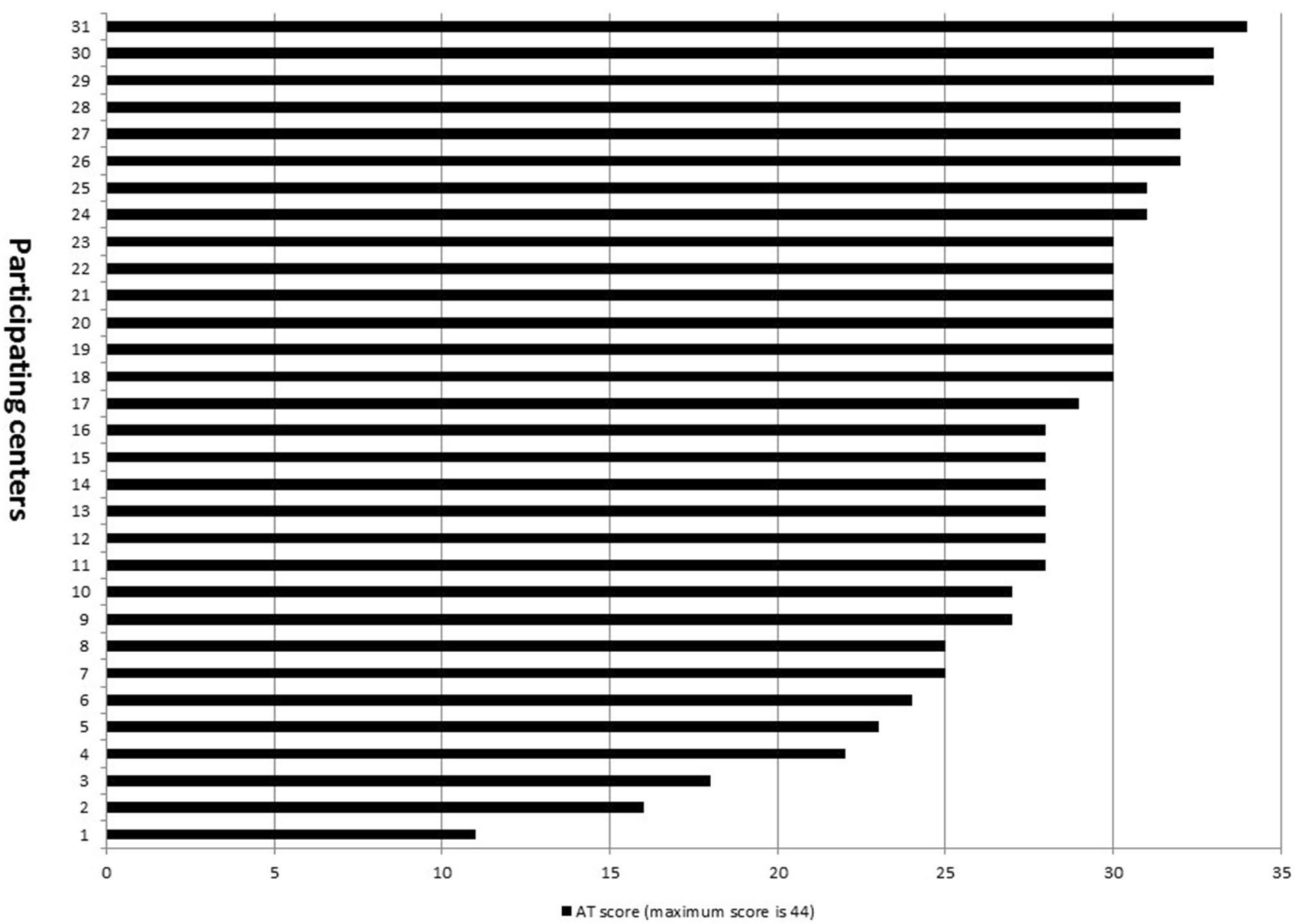

Fig. 22017 High acuity therapies (ATs) scores. AT high acuity therapy

options on their ACCU. Since a founding principle of the $\mathrm{PAC}^{3}$ is on transparent sharing, clinical champions were able to contact the specific centers who offered the types of therapy they wanted to allow on their ACCU. In contrast, fewer centers offered tracheostomy with or without oxygen in 2019 compared to 2017 . This may be explained by the fact that more centers reported utilization of more advanced tracheostomy care options including with intermittent home ventilator requirement (e.g., night only) or with continuous home ventilator ( $24 \mathrm{~h} /$ day), although the last two were not statically significant.

Over the 2-year period 13/26 centers had an increase in their overall AT score but 12/26 had a decrease. This needs further study and analysis. We understand that for any one ACCU to provide a therapeutic option, there needs to be acceptance by nursing, physician and administration leadership. Balancing patient safety and providing adequate staffing impact whether one center decides to offer a certain therapy.

The 2017 and 2019 AT scores were significantly different between the highest surgical volume group and the lowest surgical volume group. We identified therapies that were more likely to be found among centers with highest surgical volume than among centers with lowest surgical volume as shown in Table 2. Further work is needed to see if individual centers can adopt some, if not all, of these therapeutic options based on their ACCU culture, staffing and system. Allowing higher acuity therapies may be associated with improved patient care. Hart et al. found that higher acuity therapy on the ACCU was associated with shorter than expected hospital length of stay (LOS) [7].

The PAC ${ }^{3}$ is a founding member of Cardiac Networks United (CNU), a group of five learning networks partnering to improve outcomes for children and families impacted by pediatric and congenital cardiovascular disease [8]. Describing variation in care across member institutions provides an opportunity to standardize care by participating centers. Standardization of therapy has been shown previously to improve patient care $[9,10]$. With the creation of the PAC $^{3}$ registry, we plan to assess which AT elements have the highest impact on reducing LOS, minimizing complications, decreasing return admissions to the intensive care unit, and 
Table 1 High acuity therapies (ATs) items

\begin{tabular}{|c|c|c|c|c|}
\hline Therapy or Resource & Subtype & $2019 n(\%)$ & $2017 n(\%)$ & $p$-value \\
\hline \multirow[t]{4}{*}{ Inotropic support } & Milrinone & $23(88)$ & $21(81)$ & 0.703 \\
\hline & Dopamine & $12(46)$ & $10(38)$ & 0.779 \\
\hline & Dobutamine & $6(23)$ & $10(38)$ & 0.368 \\
\hline & Epinephrine & $1(4)$ & $2(8)$ & 1 \\
\hline \multirow[t]{3}{*}{ Pulmonary vasodilator therapy } & Intravenous continuous pulmonary vasodilator therapy & $18(72)$ & $18(69)$ & 1 \\
\hline & Inhaled prostacyclin (e.g., Iloprost) & $10(40)$ & $11(42)$ & 1 \\
\hline & Subcutaneous prostacyclin (e.g., Remodulin) & $17(68)$ & $16(62)$ & 0.771 \\
\hline \multirow[t]{4}{*}{ Rhythm therapy } & Antiarrhythmic intravenous infusions (e.g., esmolol, procainamide) & $6(23)$ & $7(27)$ & 1 \\
\hline & Temporary pacer wires capped & $24(92)$ & $23(88)$ & 1 \\
\hline & Temporary pacing wires with back-up pacing & $13(50)$ & $13(50)$ & 1 \\
\hline & Temporary pacing wires with pacer-dependent rhythm & $4(15)$ & $5(19)$ & 1 \\
\hline \multirow[t]{2}{*}{ Anticoagulation } & Subcutaneous enoxaparin & $25(96)$ & $24(92)$ & 1 \\
\hline & Intravenous heparin & $25(96)$ & $23(88)$ & 0.61 \\
\hline \multirow[t]{2}{*}{ Immune therapy } & Intravenous immune globulin & $24(92)$ & $26(100)$ & 0.49 \\
\hline & Immune modulators & $22(85)$ & $21(81)$ & 1 \\
\hline \multirow[t]{6}{*}{ Central line } & Peripherally Inserted Central Catheter line & $25(96)$ & $26(100)$ & 1 \\
\hline & Subclavian central venous line & $17(65)$ & $18(69)$ & 1 \\
\hline & Femoral central venous line & $17(65)$ & $20(77)$ & 0.541 \\
\hline & Umbilical venous catheter & $4(15)$ & $3(12)$ & 1 \\
\hline & Broviac central venous line & $24(92)$ & $23(88)$ & 1 \\
\hline & Transhepatic line & $6(23)$ & $6(23)$ & 1 \\
\hline \multirow[t]{5}{*}{ Drains } & Chest tubes to suction & $24(92)$ & $26(100)$ & 0.49 \\
\hline & Chest tubes to water seal & $24(92)$ & $25(96)$ & 1 \\
\hline & Mediastinal tubes & $23(88)$ & $20(77)$ & 0.465 \\
\hline & Pericardial drain & $25(96)$ & $24(92)$ & 1 \\
\hline & Pleural drains & $24(92)$ & $25(96)$ & 1 \\
\hline \multirow[t]{3}{*}{ Dialysis } & Plasmapheresis & $10(38)$ & $11(42)$ & 1 \\
\hline & Hemodialysis & $10(38)$ & $11(42)$ & 1 \\
\hline & Peritoneal dialysis & $15(58)$ & $11(42)$ & 0.406 \\
\hline \multirow[t]{8}{*}{ Respiratory support } & Nasal cannula oxygen, $100 \%$ & $25(96)$ & $25(96)$ & 1 \\
\hline & High flow nasal cannula, Initiation & $15(58)$ & $15(58)$ & 1 \\
\hline & CPAP/BiPAP initiation & $19(73)$ & $4(15)$ & $<0.001$ \\
\hline & CPAP/BiPAP for sleep only & $3(12)$ & $21(81)$ & $<0.001$ \\
\hline & CPAP/ BiPAP, continuous & $22(85)$ & $14(54)$ & 0.034 \\
\hline & Tracheostomy present, with or without oxygen & $11(42)$ & $21(81)$ & 0.009 \\
\hline & $\begin{array}{l}\text { Tracheostomy, intermittent home ventilator requirement (e.g., night } \\
\text { only) }\end{array}$ & $14(54)$ & $11(42)$ & 0.579 \\
\hline & Tracheostomy, home ventilator $24 \mathrm{~h} /$ day & $11(42)$ & $9(35)$ & 0.776 \\
\hline \multirow[t]{4}{*}{ Sedation } & $\begin{array}{l}\text { Intravenous benzodiazepines, intermittent dosing for anxiolysis/with- } \\
\text { drawal etc }\end{array}$ & $23(88)$ & $25(96)$ & 0.61 \\
\hline & Intravenous benzodiazepines, intermittent dosing for acute sedation & $10(38)$ & $12(46)$ & 0.779 \\
\hline & Intravenous narcotic pain medication, intermittent dosing & $24(92)$ & $26(100)$ & 0.49 \\
\hline & Intravenous narcotic pain medication via PCA & $23(88)$ & $25(96)$ & 0.61 \\
\hline \multicolumn{2}{|c|}{ Prostaglandin, to support pulmonary or systemic circulation } & $9(35)$ & $8(31)$ & 1 \\
\hline \multicolumn{2}{|c|}{ Anti-neoplastic } & $11(42)$ & $15(58)$ & 0.406 \\
\hline \multicolumn{2}{|l|}{ Ventricular assist device (any type) } & $19(73)$ & $15(58)$ & 0.382 \\
\hline
\end{tabular}

$B i P A P$ bi-level positive airway pressure, $C P A P$ continuous positive airway pressure, $P C A$ patient-controlled-analgesia 


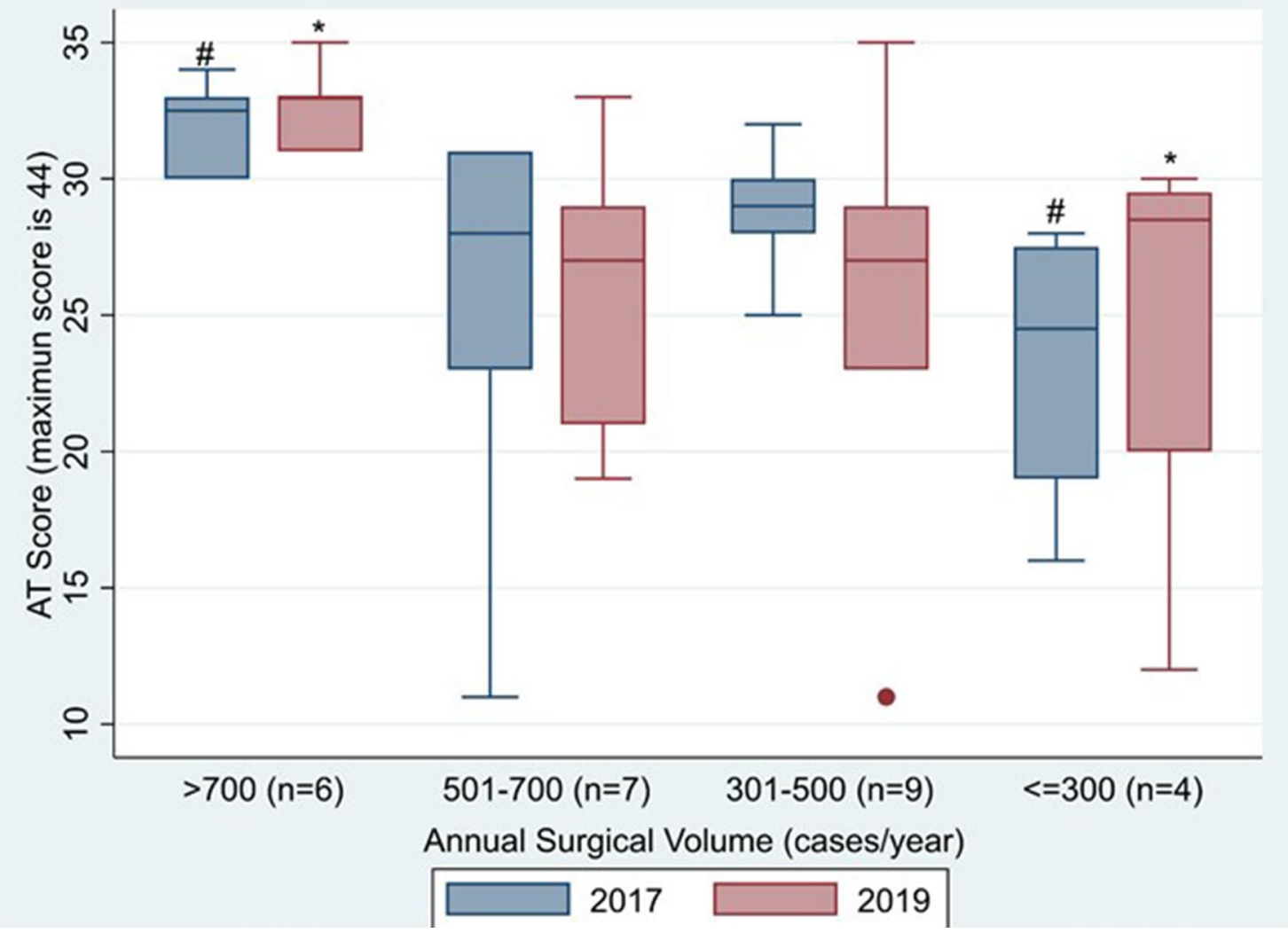

Fig. 3 High acuity therapies (ATs) across member institutions as a function of surgical volume. "Significant difference $(p=0.009)$ was noted between the highest and lowest surgical volume groups for
2017 AT score. * Significant difference $(p=0.046)$ was noted between the highest and lowest surgical volume groups for 2019 AT score. AT high acuity therapy
Table 2 Elements more likely to be found among centers with highest surgical volume compared to lowest surgical volume in 2019

\begin{tabular}{|c|c|c|}
\hline \multirow[t]{2}{*}{ Subtype } & \multicolumn{2}{|l|}{2019} \\
\hline & $\begin{array}{l}\text { Highest surgical volume } \\
(n=6)\end{array}$ & $\begin{array}{l}\text { Lowest surgical } \\
\text { volume }(n=4)\end{array}$ \\
\hline Inhaled prostacyclin (e.g., Iloprost) & $4(67 \%)$ & $0(0)$ \\
\hline Ventricular assist device & $6(100 \%)$ & $1(25 \%)$ \\
\hline Peritoneal dialysis & $4(67 \%)$ & $2(50 \%)$ \\
\hline Temporary pacer wires capped & $6(100 \%)$ & $2(50 \%)$ \\
\hline Temporary pacing wires with back-up pacing & $5(83 \%)$ & $1(25 \%)$ \\
\hline Tracheostomy present, with or without oxygen & $5(83 \%)$ & $1(25 \%)$ \\
\hline $\begin{array}{l}\text { Intravenous benzodiazepines, intermittent dosing for anxi- } \\
\text { olysis/withdrawal etc }\end{array}$ & $5(83 \%)$ & $2(50 \%)$ \\
\hline
\end{tabular}

improving patient/lesion specific clinical outcomes. Once established, therapies and practices that have a meaningful impact on outcomes can be a focus of future quality improvement (QI) collaboration among PAC $^{3}$ members. The PAC $^{3}$ community has successfully implemented QI projects that take into consideration individual center's culture and environment. Case in point is the post-operative chest tube practice project, which can be a model of how to translate registry-driven data into improvement action [11].

\section{Limitations}

This paper is based on the PAC ${ }^{3}$ hospital surveys and is not yet linked to the ACCU-specific registry database launched in February 2019. In the future, we plan to assess which AT elements are associated with highest quality-driven care in the ACCU. Also, since $\mathrm{PAC}^{3}$ and Pediatric Cardiac Critical Care Consortium $\left(\mathrm{PC}^{4}\right)$ are partner registries of the $\mathrm{CNU}$, we will be able to study clinical outcomes of our cardiac 
Table 3 Contributing Centers

\begin{tabular}{ll}
\hline $\begin{array}{l}\text { Boston Children's Hospital } \\
\text { Children's Healthcare of Atlanta/Emory University }\end{array}$ & Monroe Carell Jr. Children's Hospital at Vanderbilt \\
Children's Hospital Colorado & Nationwide Children's Hospital \\
Children's Hospital of Philadelphia & Nemours/Alfred I. duPont Hospital for Children \\
Children's Hospital of Pittsburgh & Primary Children's Hospital \\
Children's Hospital of Wisconsin & Rainbow Babies and Children's Hospital \\
Children's Mercy Hospital-Kansas City & Seattle Children's Hospital \\
Children's National Hospital & St. Louis Children's Hospital \\
Children's of Alabama (UAB) & Texas Children's Hospital \\
Cincinnati Children's Hospital & The Hospital for Sick Children \\
Cohen Children's Medical Center & University of California-San Francisco Benioff Children's Hospital \\
Lucile Packard Children's Hospital at Stanford & C.S. Mott Children's Hospital University of Michigan \\
Medical University of South Carolina & University of Virginia \\
\hline
\end{tabular}

Bold items denote authorship

patients across the continuum and correlate that with AT elements, nurse/patient ratio, and staffing models.

\section{Conclusion}

Variation in therapy within the ACCU among participating $\mathrm{PAC}^{3}$ institutions exists and presents an opportunity for shared learning across the collaborative. Respiratory therapies in ACCUs among participating centers increased from 2017 to 2019. Further study using outcomes data from the collaborative database is necessary to identify which therapies in the ACCU impact the quality and outcomes of pediatric acute cardiac care.

Acknowledgements The authors are grateful for all site champions who participated in the $\mathrm{PAC}^{3}$ hospital survey (Table 3), for Dr Angela J Doty and Lindsay Attaway for their editorial assistance, and Jiji Jiang for help with statistical analysis. The authors are also grateful for Courtney Strohacker and Dora O'Neil for their involvement in survey design and poster revision.

Author Contributions ASH was involved in study design, analysis of data, and drafting the manuscript. AKK was involved in survey design, interpretation of the study findings, and execution and revising the manuscript. SAH was involved in the analysis and interpretation of the study findings, reviewing draft with critical and substantial input. SCC was involved in survey design, interpretation of the study findings, and revising the manuscript. MLC was involved in survey design, interpretation of the study findings, and revising the manuscript. AMH was involved in survey design, interpretation of the study findings, and revising the manuscript. AKH was involved in survey design, interpretation of the study findings, and revising the manuscript. MG was involved in the creation and management of the REDCap survey database, interpretation of the study findings, and in revising the manuscript. NLM was involved in survey design, interpretation of the study findings, and revising the manuscript. AMB was involved in interpretation of the study findings and revising the manuscript. ÉDG was involved in interpretation of the study findings and revising the manuscript. SSP was involved in study design, interpretation of the study findings, and revising the manuscript. JEB was involved in statistical analysis and revising the manuscript. RET was involved in the study design, interpretation of the study findings, and in revising the manuscript. This submission is with the full knowledge and approval of the listed coauthors.

Funding All authors have reported that they have no relationships relevant to the contents of this paper to disclose. The PAC ${ }^{3}$ is supported by the Heart Institute at Cincinnati Children's Hospital, Cardiac Networks United, and $\mathrm{PAC}^{3}$ member institutions.

\section{Declarations}

Conflict of interest The authors have no conflict of interest relevant to this article to disclose.

Ethical Approval Cincinnati Children's Hospital Medical Center Institutional Review Board approved this study with a waiver of consent granted.

\section{References}

1. Kipps AK, Cassidy SC, Strohacker CM et al (2018) Collective quality improvement in the paediatric cardiology acute care unit: establishment of the Pediatric Acute Care Cardiology Collaborative (PAC3). Cardiol Young 28(8):1019-1023

2. Willis AJ, Hoerst A, Hart SA et al (2020) The added value of the advanced practice provider in paediatric acute care cardiology. Cardiol Young 31:1-4

3. Hoerst A, Bakar A, Cassidy SC et al (2019) Variation in care practices across pediatric acute care cardiology units: results of the Pediatric Acute Care Cardiology Collaborative (PAC (3)) hospital survey. Congenit Heart Dis 14(3):419-426

4. Mott AR, Neish SR, Challman M, Feltes TF (2017) Defining pediatric inpatient cardiology care delivery models: a survey of pediatric cardiology programs in the USA and Canada. Congenit Heart Dis 12(3):294-300

5. Harris PA, Taylor R, Thielke R, Payne J, Gonzalez N, Conde JG (2009) Research electronic data capture (REDCap)—a 
metadata-driven methodology and workflow process for providing translational research informatics support. J Biomed Inf 42(2):377-381

6. Harahsheh AS, Ottolini M, Lewis K, Blatt B, Mitchell S, Greenberg L (2016) An innovative pilot curriculum training pediatric residents in referral and communication skills on a cardiology rotation. Acad Pediatr 16(7):700-702

7. Hart SA, Tanel RE, Kipps AK et al (2020) Intensive care unit and acute care unit length of stay after congenital heart surgery. Ann Thorac Surg 110(4):1396-1403

8. Gaies M, Anderson J, Kipps A et al (2018) Cardiac Networks United: an integrated paediatric and congenital cardiovascular research and improvement network. Cardiol Young 29:1-8

9. Harahsheh AS, Hom LA, Clauss SB et al (2016) The impact of a designated cardiology team involving telemedicine home monitoring on the care of children with single-ventricle physiology after norwood palliation. Pediatr Cardiol 37(5):899-912

10. Anderson JB, Beekman RH 3rd, Kugler JD et al (2015) Improvement in interstage survival in a national pediatric cardiology learning network. Circ Cardiovasc Qual Outcomes 8(4):428-436

11. Bates KE, Madsen NL, Khadr L et al (2020) Center variation in chest tube duration and length of stay after congenital heart surgery. Ann Thorac Surg 110(1):221-227

Publisher's Note Springer Nature remains neutral with regard to jurisdictional claims in published maps and institutional affiliations. 